Original article

\title{
The voice of Indian women on family planning: A qualitative systematic review
}

\author{
Shradha S. Parsekar ${ }^{a}$, Praveen Hoogar ${ }^{\text {b, }}$, Vijay Shree Dhyani ${ }^{\text {a }}$, Uday Narayan Yadav $^{c}$ \\ ${ }^{a}$ Public Health Evidence South Asia, Department of Health Information, Prasanna School of Public Health, Manipal Academy of Higher Education, Manipal, India \\ ${ }^{\mathrm{b}}$ Centre for Bio Cultural Studies, Directorate of Research, Manipal Academy of Higher Education, Manipal, India \\ ${ }^{c}$ Centre for Primary Health Care and Equity, Faculty of Medicine, University of New South Wales, Sydney, Australia
}

\section{A R T I C L E I N F O}

\section{Keywords:}

Contraception

Family planning

India

Qualitative systematic review

\begin{abstract}
A B S T R A C T
Objective: The systematic review explores personal experiences and perceptions of (and on) resident Indian women on family planning.

Methods: We included qualitative studies conducted among women, husbands and mothers-in-law. Search on five databases (published between 2000 and May 8, 2019) and forward and backward citations search of included studies were undertaken (April 2020). Three-stage screening and data extraction were done independently. Iterative process was followed to thematically analyse the data and presented it using the World Health Organization conceptual framework on social determinants of health.

Result: Of 857 citations, we included 48 qualitative studies. Women's intent of using family planning services/ methods was influenced by multiple factors such as socio-demographic characteristics. Women's agency and social status affected their standing in family planning decision-making. Fear of side-effects of contraception methods, access to information and support, and cultural and religious beliefs hindered the use of modern methods of contraceptive. Undesirable attitudes of service providers influenced underutilisation of family planning services. Ineffective contraceptive choice counselling, particularly for poor section of the community and high-risk groups were reported. While considering inter-pregnancy interval, some of the women opted for induced abortion to terminate unplanned pregnancy however, had meagre acceptance for using modern contraceptive methods.

Conclusion: The findings call for reinvigorated efforts to provide effective and individually, religiously, regionally and culturally appropriate interventions to improve family planning access and ultimately support better health and rights of women in India.
\end{abstract}

\section{Introduction}

India, a densely and second highest populated country in the world, is in a phase of demographic and epidemiological transition. ${ }^{1}$ To control its rising population, India felt an urgency of Family Planning (FP) program in 1952 and became one of the first countries to launch the "National Programme for Family Planning" (NPFP). Since its launch, the NPFP has undergone various policy and implementation modifications and currently operating as part of Reproductive, Maternal, Newborn and Child Health and Adolescents Program (RMNCAH+). ${ }^{2}$ In the initial period of NPFP, the emphasis was on natural methods (e.g., rhythm method); subsequently the focus shifted on modern methods of contraception such as condoms, diaphragms, and intra-uterine devices
(IUDs). Between 1962 and 1977, India adopted target-based, mass sterilisation, monetary incentives and other coercive methods (e.g., male sterilisation). ${ }^{3}$ In 1977, voluntary efforts of population control, extending the services to maternal and child health $(\mathrm{MCH})$ and incorporating indirect methods of population control were executed. Consequently, the focus shifted on addressing the unmet needs of contraception. ${ }^{3}$ Furthermore, India is now devoted to global initiatives that focus on the Sexual and Reproductive Health (SRH) and rights ${ }^{4-6}$ and Sustainable Development Goals (SDGs) e.g., goal no. 5 ("gender equality and empowerment of women/girl") and target no. 3.7 (ensuring universal access to SRH services). ${ }^{4}$ Likewise, in 2012, India obliged to achieving FP2020 (now FP2030) goals of improving access, choice, and quality of FP services. ${ }^{7}$

\footnotetext{
* Corresponding author. 576104, Karnataka India.

E-mail address: praveen.hoogar@manipal.edu (P. Hoogar).
} 
Over the years, India has made progress in FP-related indicators (e. g., reduction in fertility rate from $4.97 \%$ in 1980 to $2.44 \%$ in 2015 ). ${ }^{3}$ Despite this achievement, India is still distant in achieving some of the targets laid down by the SDG (i.e., satisfying the $75 \%$ of the total demand by use of modern FP methods). ${ }^{4}$ The unmet FP needs among Indian women especially belonging to lower socio-economic strata are high. ${ }^{8}$ About $54 \%$ married and $44 \%$ all women (15-49 years) use any type of contraceptive, and $48 \%$ married and $38 \%$ all women (15-49 years) use modern contraceptive methods. ${ }^{9}$ Among these women, within one year of use, the discontinuation rate of contraceptives was high, ${ }^{10}$ which are related to multiple factors. ${ }^{8}$ Compared to male sterilisation and temporary modern contraceptives, female sterilisation (accounting to $37 \%$ of women between 15 and 49 years of age), is the most preferred approaches of limiting the family size. The number of female sterilisations carried out per annum is also higher as compared to other countries. ${ }^{8}$ In 2015, the unintended pregnancy rate in India was 70.1 per 1000 women (15-49 years) and one-third of all the pregnancies ended in induced abortions. Furthermore, the health facility survey conducted in six Indian states reported that there were 15.6 million abortions with a rate of 47 abortions per 1000 women in reproductive age group. ${ }^{11},{ }^{12}$

By addressing FP needs, more than $90 \%$ of low- and middle-income country's child deaths and morbidity associated with abortion and pregnancy could be avoided. ${ }^{5},{ }^{11},{ }^{13-15}$ Considering this, the government of India is committed to enhancing the demand for modern reversible contraceptives use to $54.3 \%$ among married women (15-49 years). ${ }^{9},{ }^{10}$

To highlight commonalities (or dissimilarities), it is important to collate and summarise the opinion and perspectives of people belonging to a wider group or context on a complex phenomenon. To the best of our knowledge, there are no qualitative systematic reviews conducted on the resident Indian population that studied perceptions of FP. Previous qualitative systematic reviews conducted elsewhere were focused on a subset of population such as age group, provider or country. ${ }^{6},{ }^{16-19}$ Therefore, the present qualitative systematic review was designed to explore personal experiences and perceptions of and on women about FP in India. This review will fill the gap by accounting for the experiences, expectations, emotions, needs, opinions, and feelings of resident Indian women on the FP, which will further help guide policies and frame FP interventions in India.

\section{Material and methods}

The protocol for this systematic review was registered with "PROSPERO: International prospective register of systematic reviews" (CRD42018093960). The Preferred Reporting Items for Systematic Review and Meta-analysis has been adhered.

\subsection{Inclusion and exclusion criteria}

Detailed inclusion and exclusion criteria are reported in Table 1.

\subsection{Searching studies}

Electronic databases viz. Medline (PubMed and Web of Science), CINAHL, Scopus, and ProQuest were searched with a tailored search strategy for each database until May 8, 2019. Multiple factors influence FP and as time passes the perceptions may change hence, we decided to consider studies that were published in the year 2000 or after (this restriction was not imposed at the time of protocol). Additionally, reference lists and forward citations (on April 12, 2020) of all included studies were searched manually to find any relevant publications. The search strategy (Additional file) combined keywords such as 'Family Planning', 'Contraception', 'Experiences', 'Perception', 'Focus Group Discussion', 'In-depth interviews', 'Ethnography'.
Table 1

Inclusion and exclusion criteria.

\begin{tabular}{|c|c|}
\hline Types of studies & $\begin{array}{l}\text { The studies focused on qualitative data including, but not } \\
\text { limited to, phenomenology, ethnography, action research and } \\
\text { grounded theory were eligible to be included. We considered } \\
\text { qualitative data collection methods such as in-depth } \\
\text { interviews, focus group discussions, observations and } \\
\text { qualitative case studies. Additionally, descriptive qualitative } \\
\text { studies that studied the experience or perceptions were } \\
\text { included. In case of mixed methods studies, only results of } \\
\text { qualitative analysis were considered. }\end{array}$ \\
\hline $\begin{array}{l}\text { Phenomena of } \\
\text { interest }\end{array}$ & $\begin{array}{l}\text { Studies that probed the experiences and/or opinions on family } \\
\text { planning and related issues (such as barriers or facilitators of } \\
\text { contraception use) were included. For the purpose of this } \\
\text { systematic review, family planning was operationalised (in } \\
\text { accordance with the World Health Organization) as a } \\
\text { conscious effort of couple to limit childbirths or have } \\
\text { appropriate inter-pregnancy interval using temporary or } \\
\text { permanent contraceptive methods. Additionally, we } \\
\text { considered studies that discussed treatment of involuntary } \\
\text { infertility. }\end{array}$ \\
\hline $\begin{array}{l}\text { Population and } \\
\text { context }\end{array}$ & $\begin{array}{l}\text { Women (irrespective of age and fertility status) in } \\
\text { reproductive age groups living in India were included. } \\
\text { Additionally, perception of husbands, mothers and mothers- } \\
\text { in-law; if provided in the study were captured to address } \\
\text { pluralism. Men or mothers-in-law's opinion supplemented the } \\
\text { information in generating the similarities or dissimilarities in } \\
\text { women's opinion. Nevertheless, we excluded the study if it } \\
\text { was exclusively focused on men or healthcare providers. In the } \\
\text { case of a mixed population, we extracted perceptions of the } \\
\text { population of our interest. Additionally, we considered studies } \\
\text { undertaken on people living with HIV, drug users, and women } \\
\text { undergoing abortion, if it was focused on phenomena of our } \\
\text { interest or had described at least one theme/subtheme of } \\
\text { family planning. Studies conducted in other countries among } \\
\text { Indian women such as American Indian were excluded. }\end{array}$ \\
\hline Publication type & $\begin{array}{l}\text { Journal publications, theses, dissertations, reports, books or } \\
\text { book chapters were included. We included studies irrespective } \\
\text { of publication type or peer reviewed status. However, reviews, } \\
\text { secondary analyses, editorials, commentaries, letters, and } \\
\text { conference proceedings were excluded. }\end{array}$ \\
\hline Language & We included studies published in English. \\
\hline
\end{tabular}

\subsection{Selection of studies}

EndNote $\times 7$ was used to manage the data and Microsoft Excel spreadsheet for screening. Two authors (PH, SSP) screened citations by their titles and abstracts, independently. Full-text screening was undertaken independently by PH, SSP, and VSD in two groups. Any disagreements between the authors on a decision to include the articles were resolved by discussions until consensus within and between groups.

\subsection{Data extraction}

A data extraction sheet was prepared, and extraction was carried out by PH, SSP, and VSD, independently, in pairs. Disagreements, if any, on extracted data were resolved by discussions, within and between the pairs. The extracted data included study identifiers, data collection methodology, study location, contextual details of participants, and qualitative data of phenomena (themes and quotes).

\subsection{Data analysis}

We undertook manual thematic analysis to analyse the qualitative data. ${ }^{6},{ }^{16},{ }^{20},{ }^{21}$ SSP read the extracted data multiple times and generated the coding frame. Quotations reported in included studies were read and deciphered to get a deeper understanding of the data. Each study was examined for consistencies, dissimilarities and contradictions in the perceptions or experiences to generate a new, wider understanding of the phenomena, without altering the originality of the primary data. 
The coding scheme enabled the systematic identification of analytic patterns and theoretically important concepts. SSP and PH further discussed these codes, refined them (by rereading the study, if needed) and grouped the codes after reaching consensus. Subsequently, repeated occurring codes were synthesised and classified into overarching themes, which led to the development of thematic framework. Within each theme we described the content and meaning of the data extracted from included studies. To confirm trustworthiness, the emerging themes were re-examined by all the authors. Subsequently, we prepared the final list of themes through discussion and consensus. A theme appearing in more than one paper does not justify the intensity of data validity. Therefore, study references within a specific theme are reported. To summarise and explain the linkages between the themes and subthemes we utilised the "World Health Organization (WHO) conceptual framework on the social determinants of health". ${ }^{22}$

\section{Results}

\subsection{Included and excluded studies}

This systematic review included 48 studies for analysis. The threestage study selection process and reasons for exclusion are documented in Fig. 1. The list of included studies, with citations and its characteristics can be found in additional file. Included studies were conducted in 14 Indian states having regional representation (Fig. 2). In addition to women, men's ( $\mathrm{n}=16$ studies), mothers-in-law or older women's $(n=7)$ and key informant's $(n=12)$ viewpoint was captured. Key informants were community members, volunteers, peer supporters or traditional healers.

\subsection{Findings of the systematic review}

In accordance with the "WHO conceptual framework on the social determinants of health", ${ }^{22}$ we summarised the concept of FP in India (Fig. 3). Various complex elements that foreground the structural and intermediary determinants of FP decision-making (timing, inter-pregnancy interval and a number of children) and choice of FP methods emerged from the data. Various themes and subthemes that emerged from the data are briefly described in Table 2 and detailed synthesis can be found in the additional file.

\section{Discussion}

To our best knowledge, this qualitative systematic review is the first one conducted to collate the evidence to inform on perceptions and experiences of women about FP in India. Findings highlights that the multiple determinants influenced women's intent of using FP services or methods. These determinants can be linked to improving the unmet FP needs and SRH and rights, in conformity with the SDGs 3 and 5.

Although review participants were in favour of getting married after 18 years, some women were married, parous or pregnant before attaining the legal age of marriage (18 and 21 years for women and men in India, respectively). ${ }^{23}$ As per a national survey conducted in India, there were a significant proportion of adolescent (15-19 years) women who were married $(15.2 \%)$ and pregnant or parous $(8 \%)$ in the year 2015-16. ${ }^{11},{ }^{24}$ This is a major concern especially for India considering the effect of adolescent pregnancy and childbirth on MCH. Early marriages are often associated with higher fertility rate, early childbearing practices, shorter birth intervals, and decreased knowledge and access to FP methods. ${ }^{25}$

Marriage brings numerous responsibilities to women, and women are expected to perform in an "ideal" way as defined by societal pressure and in-laws' expectations. It is a cultural notion, especially in India, that marriage is not only a union of two people, but also a union of two families. Additionally, marriage embarks on the societal and familial expectation of initiating a childbearing and childrearing. But, soon after marriage women are at the forefront to take the entire responsibility to prove their womanhood and receive respect from the family and society by being fertile and childbearing; if not, there was a threat for experiencing discrimination and divorce.

Most of our participants were not or minimally educated and had low socio-economic status. The majority of the participants were favouring small family size and were well versed with its benefits including reduced financial burden, $\mathrm{MCH}$ and other responsibilities associated with child-rearing. The finding is congruent with existing evidence of importance of investing in FP. ${ }^{26}$ Nonetheless, almost one-fourth of the studies reported that having a son was one of the prime factors for influencing FP decision-making. Reasons for son preference were

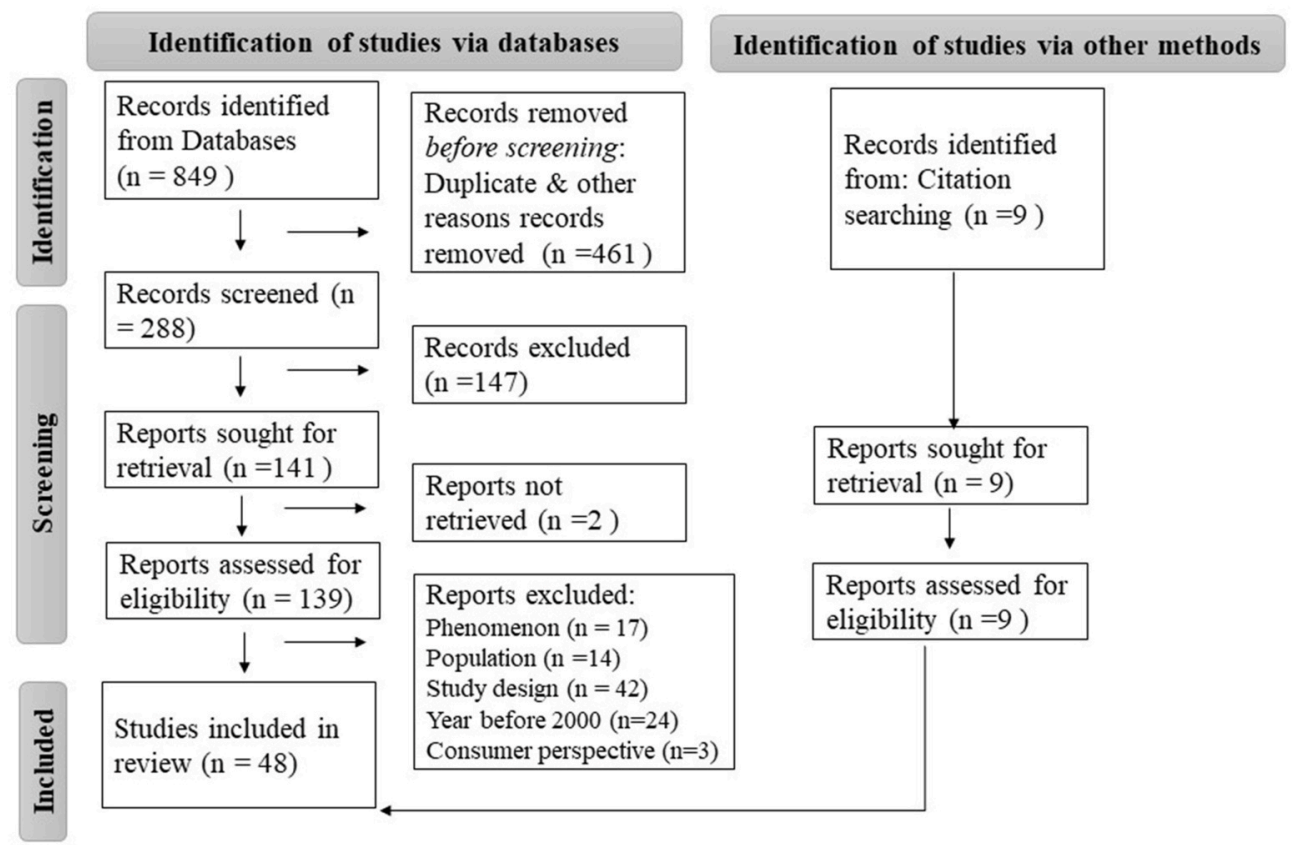

Fig. 1. PRISMA 2020 flow diagram. 

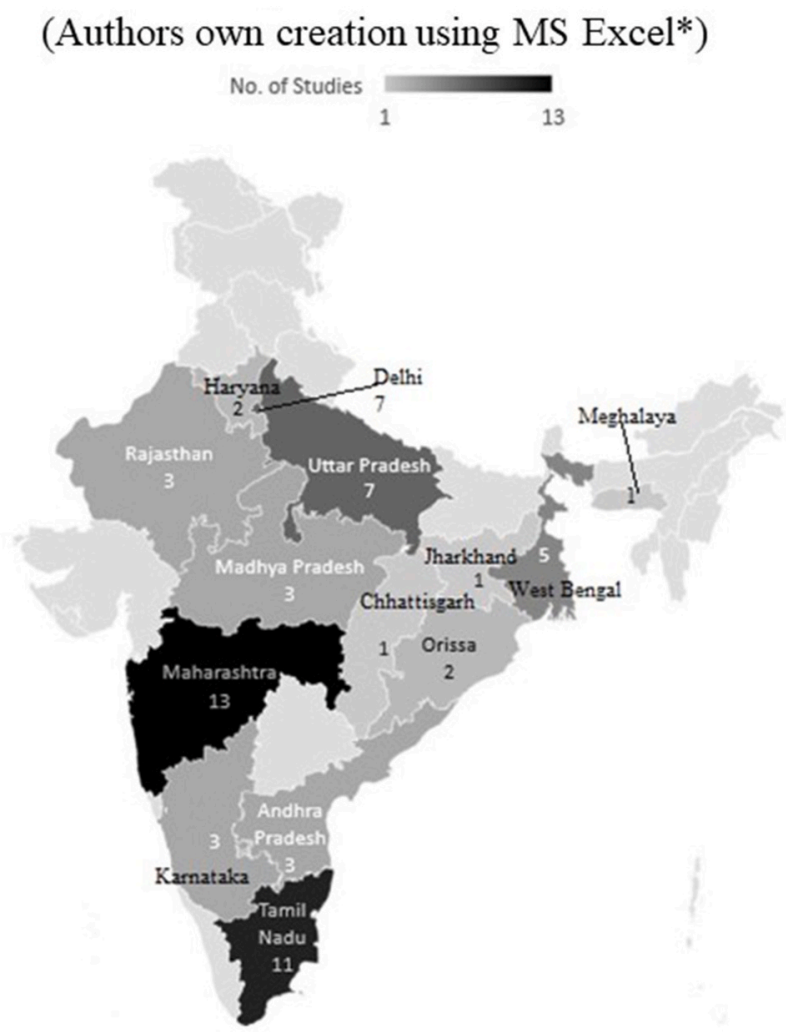

*Map was created by authors using MS Excel spreadsheet insert map chart feature on windows 10. Data (frequencies)
were entered state-wise on an Excel spreadsheet and plotted on map chart.

Fig. 2. Map of India showing state-wise distribution of included studies (Authors own creation using MS Excel*)

*Map was created by authors using MS Excel spreadsheet insert map chart feature on windows 10. Data (frequencies) were entered state-wise on an Excel spreadsheet and plotted on map chart.

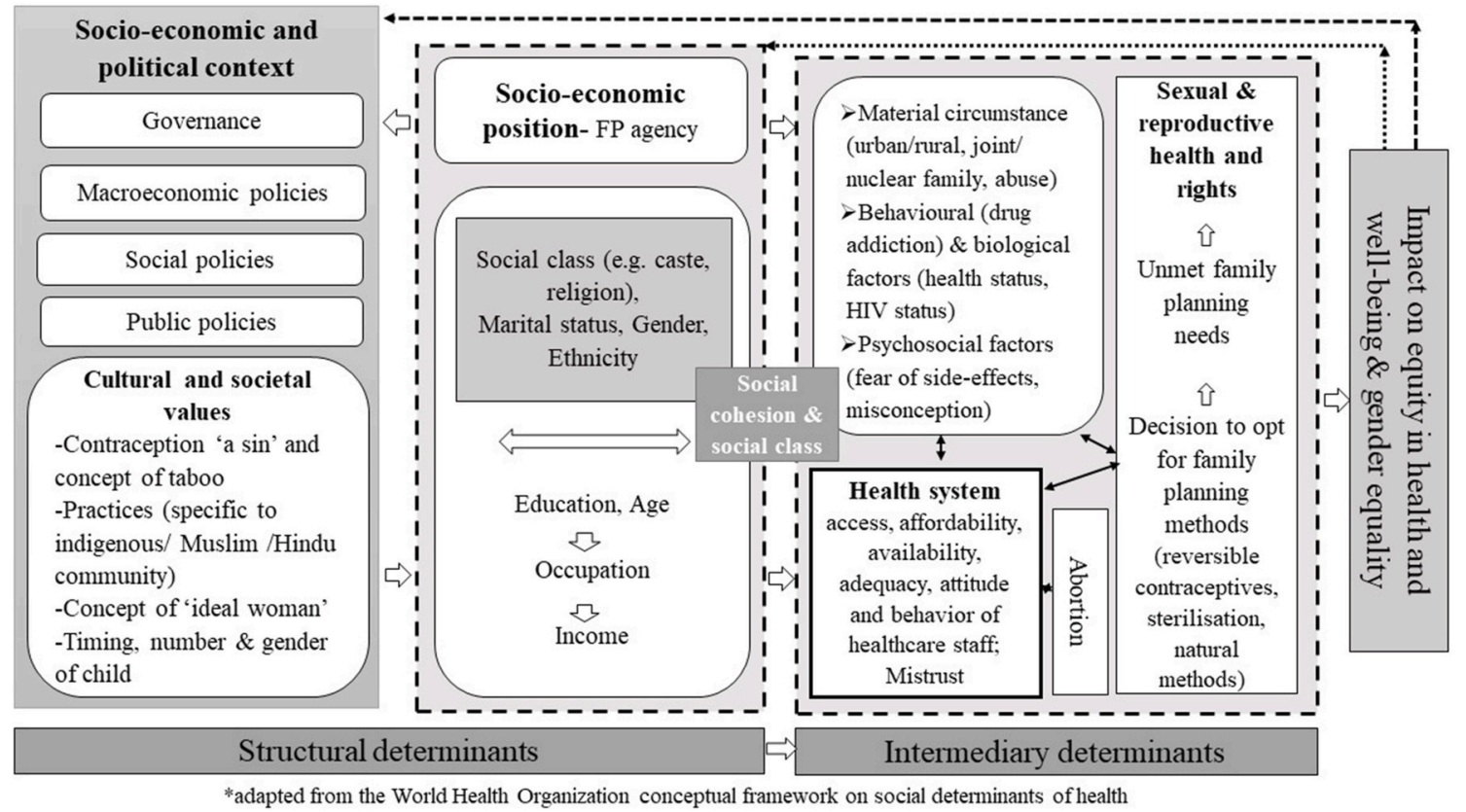

Fig. 3. Conceptual framework on family planning in India*

*adapted from the World Health Organization conceptual framework on social determinants of health.

multi-fold and deeply rooted in the culture. Participants spoke about gender selective abortions. This evidence is congruent with other literature, that suggests India and China together contributes to nine out of
10 gender selective abortion. ${ }^{25}$ Gender selective abortion reflects the manifestation of deep-rooted gender bias irrespective of socio-economic status of people in India. 
Table 2

Themes/subthemes and summarised result of perception of family planning in India.

\begin{tabular}{l} 
Themes \\
\hline 1. Structural determinants \\
1.1. Socio-economic \& political \\
context
\end{tabular}

context

1.2. Cultural and societal values

1.2.1. Marriage and sexuality

1.2.2. Social-cultural-religious norms

Subthemes/Categories

Structural factors determine stratification and division of social class within the society, and it defines person's socio-economic standing within ladder of power, prestige and access to resources. It includes context, structural mechanisms and the resultant socioeconomic position, these together influence intermediary determinants. ${ }^{22}$ Various contextual factors emerged from the data; these are described further. Detailed synthesis can be found in Additional file.

Social policies: As per government order, Baiga community in Chhattisgarh denied access to family planning (FP) services as a result, women had multiple pregnancies, which deteriorated health. Because of this, women visited nearby states to avail FP services, or they hide/gave up their cultural identity.

Some of the cultural and societal values related to FP (timing, number \& gender of child) were mythic and women/couple follow these norms willingly. However, some participants presumed that they do not have choice but to oblige societal and family pressure. Some norms are specific to indigenous, Muslim or Hindu family. Sub themes under this are described further. Women perceived the ideal age for marriage to be 18 years. After marriage, society expected woman to be 'ideal' wife and mother; and women perceived that it was their duty to fulfil these expectations. Women expressed that they were expected to be sexually available for husbands. Husband's dominance in sexuality and women's submissiveness were commonly reported. Threats from husband about re-marriage were reported. Although, polygamy among tribal was not common, it increases the fertility prospects. Also, tribal population accepted the pre-marital sex and relationships, as well as childbearing. However, discussion on sex was a taboo and need for sex education was discussed.

Women perceived that it was the social obligation to have their first child soon after marriage. There were different reported reasons for the same, viz. Familial and societal pressure, community judgement, and proving womanhood. If a woman has a child soon after marriage her social status (respect from society and family) improves. Some women also shared threat of abandonment if they don't have a child after marriage. Participants also shared consequences of infertility.

Family planning (FP) and culture: FP is culturally not acceptable in some culture. Participants were influenced by faith healers who discouraged FP. Participants avoid talking about family planning because of shame or embarrassment. Sterilisation was not acceptable and barren woman's prospect of going for levirate marriage decreased. Some other tribal practices were also discussed, which prevented women going for FP. Family planning and religion: It was found that 'people's volition in contraceptive use or sterilisation was a sin'. Participants voiced that tribes were banned from worshiping after sterilisation and Islam forbids contraception or sterilisation. They often felt that it was God's wish in deciding the number and timing of children. Some also expressed that if a woman was sterilised, she was considered 'impure for the last rites'.

Most participants expressed timing of childbearing to be soon after marriage because it gives them feeling of incompleteness. Some felt it should be
Table 2 (continued)

Themes Subthemes/Categorie

delayed and others expressed it should be depending on the age of the women. Sex composition and preference: Only a subset of women did not prefer a particular gender of the child, while others expressed to have one girl and a boy. Those who expressed girl preference felt that girls are useful for instrumental help and they get bride-price. Girl aversion was common considering the dowry practice in India. Son preference was predominantly seen and was determined by number of children, economic and social benefits, family name, and old-age support.

Concept of family size and spacing varied between studies. Small family norm was expressed considering the economic benefits, future prospects, maternal and child health. However, others felt having multiple children were useful, while some others left it on God's wish. HIV status of parents was also important predictor of deciding the family size.

1.3. Socio-economic position Women's agency (in terms of choice- having ability to opt for FP, voice- capacity to assert one's interest and power in constraining or enabling voice and choice) is influenced by multiple factors. In contrast to younger (adolescent or youth) and not/less educated women, older women are more likely to have a steady stand and decision of timing and number of children. Older women were more inclined to use permanent sterilisation methods. Furthermore, abuse (from the husband), threats of divorce/separation and remarriage made women submissive to the men's decision on FP. Male dominance, influence of mother-in-law and women's submissive behaviour were commonly reported by the included studies that were hindering the socio-economic position of women. However, it was women's responsibility to use the contraceptive methods, and there was resistance from men to use condom or male sterilisation.

1.3.1. Decision-making norms around FP

1.3.2. Education and awareness

Women who had strong premarital agency (agency here is a general concept and not restricted to FP) or who were older in age tended to have better decision-making power and they were found to delay their marriage and childbearing for career or education. Other socio-demographic factors were employment, education, age of women (described in section 1.3) and husband's employment status. Some expressed joint decision making, however, majority expressed it as male dominance. Motherin-law's involvement in FP decision making is strongly rooted in Indian culture. Participants reported female centric nature of contraception and lack of involvement of husbands in FP counselling or discussions.

Women were aware of contraceptive methods but were not aware of their proper use, and were found to be mostly using sterilisation, rhythm method and injectable. Others were not aware of reversible contraceptives other than abstinence. FP information was often sought from friends of the same gender, Accredited Social Health Activist or were influenced by healthcare providers and media. Women had limited access to FP information and expressed further need for information. Lack of awareness on FP was also found among husbands. Women's education influenced use of FP. Education and occupation are interlinked and are related to economic standing of the women. Educated women may have better access to information, which in turn help in making an informed decision-making on FP.

(continued on next page) 
Table 2 (continued)

Themes
$\begin{aligned} & \text { 1.3.3. Women's counteraction to } \\ & \text { spousal FP rejection }\end{aligned}$

spousal FP rejection

2. Intermediary determinants

2.1. Fertility intention among HIV positive or Drug users

2.2. Induced abortion and family planning

2.3. Health system

3. Family planning methods
Subthemes/Categories

Women expressed desire to use contraception, but often left with no choice. As a result, they were left with anxiety of pregnancy or tried different tactics to avoid pregnancy such as going to their natal house or subversive avoidance of sexual intercourse, coming to bed late and secretly using FP methods.

In line with the conceptual framework, we identified multiple intermediary determinants of health. Fear of side-effects of reversible contraceptives was one of the major psychological barriers of using FP methods. Health status of women or of first born, HIV status of women or husband, and history of drug addiction influenced decision on use of FP. Furthermore, access, availability of healthcare facilities and attitude of healthcare professionals were documented. Two conditions on which people were deciding to take a call on the FP methods or choices were, family size achieved and inter-pregnancy interval. When desired family size was achieved, participants either opted for sterilisation or induced abortion. Whereas, to achieve a desired inter-pregnancy interval family opted for contraceptives or induced abortion. This finding also suggests that participants opted for induced abortion to terminate unplanned pregnancy to limit the number of children or spacing between the pregnancies.

Fertility intention among HIV positive or drug users were expressed. The desired were based on existing child(dren) or son preference, pressure from society, wanted some reason to live, and positive reinforcement after antiretroviral therapy. Inconsistent condom use was reported among HIV positive individuals. Desire to have a child also depended on health of the couple, fear of HIV transmission to child and future prospects.

Women usually resorted to abortion as a means to end unintended pregnancy to limit family size, or wanted to have spacing between childbirths. Some even opted for multiple induced abortions. Reasons were first child's health or women's health, resistance to use contraception, domestic violence, economic reasons, sex selective abortion, first child male, conceived in the month of 'Adi' in Tamil Nadu, pregnant with man having same gotra (Clan), influence of astrology, and were shameful because of existing adolescent child. However, others considered abortion as sin. Abortion among unmarried was also reported. The easy accessibility of healthcare services was reported to go for abortion. Women desired confidentiality while resorting to abort the child. Some women also reported unprofessional methods of abortion.

As a result of not conceiving soon after marriage women sought advice and treatment from faith healers and unqualified healthcare providers.

Women believed in fate, spirits, witchcraft or the 'evil eye' that determined their ability to conceive. Faced embarrassment at the idea of seeking treatment for infertility. Information access, cost, availability, accessibility, waiting time, navigating healthcare facilities and unavailability of trained staff were some of the factors to avail the healthcare services. It also dependent on familiarity of healthcare staff. Participants were discouraged by false commitments or threats, indifferent attitude of healthcare providers.

The description under this subtheme is overlapped between structural and intermediary determinants.

Female sterilisation was favoured option on family
Table 2 (continued)

\begin{tabular}{ll}
\hline Themes & Subthemes/Categories \\
\hline size completion and was considered as necessary \\
and inevitable, one time, no fear of pregnancy \\
once the family size is complete. Women \\
expressed fear of side-effects, and \\
misconceptions, cumbersome to use irreversible \\
modern contraceptives. Male sterilisation was not \\
a favoured option as it was considered to cause \\
loss of vitality or impotency. Some considered \\
contraceptives or sterilisation use against their \\
religion. There was lack of information on use, \\
disposal, access and cost of condoms. \\
Participants felt condoms hinders sexual \\
pleasure, and associated it with extra-marital \\
sex. Some participants trusted traditional or \\
natural methods of birth control such as coitus \\
interruptus, rhythm method, abstinence, and sex \\
during menstruation. These methods were \\
encouraged by faith healers and were simple, \\
healthy, and economical, and had no side- \\
effects.
\end{tabular}

Our review participants comprehended inter-pregnancy spacing intervals, however intention of using contraceptives was not corresponded. One of the major decision-making factors for FP use was husband's support, but regrettably most of the women received meagre support from husbands and mothers-in-law. Majority of the women complied with the husband's decision, but some women took the risk of going against the family's decision and secretly resorted to using contraceptives and as a result lived with a fear of getting caught. Women remain at socially disadvantaged positions due to gender inequality and lack of decision-making power (related to number and timing of childbirth). ${ }^{24}$ FP not only addresses the unmet contraceptive needs, but also helps in overcoming gender equity and equality, and growth of the individual, society and country. ${ }^{11}$

There were misconceptions about using FP methods (in general) across the country, disapproval regarding religious and socio-cultural norms and paradox related to men's attitude. Similar findings were reported by reviews conducted among $40+{ }^{19}$ and younger than 25 years aged women. ${ }^{6}$ Furthermore, we found that female sterilisation was the most utilised method of contraception once the desired family size was reached. Aforesaid finding was consistent with Indian data, wherein female sterilisation accounted for three out of four modern contraceptive uses. ${ }^{11}$ Women's preference to use sterilisation could be due to fear of using reversible methods of contraception and partly because of India's history of FP programs. ${ }^{9}$ Indian FP program, through its inception, mostly focused on limiting the family size by encouraging women to adopt permanent sterilisation methods. Additionally, history of coercive male sterilisation during 70 s might have created a lasting fear among the people. Although the program received multiple modifications, it is deeply rooted in people's mind, which may be the reason for favouring female sterilisation. Furthermore, high female sterilisation might be related to associated reasons of not favouring temporary modern contraceptives as described below.

Our review demonstrated the difficulties in complying with oral contraceptive pill (OCP) regimen. Participants associated the use of condoms with extra marital sex, commercial sex and sexual transmitted diseases, which hindered its use. Women had lack of agency or control over condom use as it was considered a male contraceptive. Almost all the studies reported fear of side-effects of contraception (due to prior experience or heard from others), especially IUDs and OCPs, therefore they were inclined to use traditional methods of contraception. Similar findings were reported by other reviews. ${ }^{6},{ }^{25}$ In 2015 , there were about $13 \%$ Indian women who had unmet contraceptive needs and $6 \%$ women practiced traditional methods of contraception. ${ }^{12}$

Some of our review participants opted for induced abortion for unplanned pregnancy as a result of unmet FP. Inclination for induced 
abortion could be due to multiple factors such as accessibility to its use, ${ }^{6}, 11,25$ resistance to modern contraception methods (as discussed above) or lack of awareness related to FP. Our findings are compatible with quantitative studies conducted in India ${ }^{11},{ }^{12}$ and further provide the perceptions of women for its inclination. As discussed in the background, induced abortion is associated with adverse health condition (as well as social stigma due to which many women hide abortion history).

Our review highlights the FP service use among drug addicts and PLHIV. The possible reason behind this could be effective contraceptive choice counselling among high-risk populations for preventing sexually transmitted infections. However, the findings based on the general population reported that condom use was considered as a barrier to sexual pleasure. This underscores the urgent need of a health literacy program by developing both the skills and mind-set about its proper use. $^{27}$ One of the included studies in our review reported the atrocities faced by indigenous people in Chhattisgarh due to FP policy change (government order 1979). Nevertheless, it is important to note that Chhattisgarh High Court quashed this order in 2018 as the order was violating human rights. ${ }^{28}$

In general, our review participants had credibility issues on government health facilities due to various reasons, such as the attitude of the healthcare professionals, which is coherent with the conflict theory of decision-making. ${ }^{29}$ Similar to our findings, accessibility, economic reasons, social stigma and prejudices of healthcare providers were the main reason for not accessing FP services. However, not utilising professional healthcare services eventually intensifies the risk of medical complications. ${ }^{11}{ }^{16}$ Gender of the attending doctor was found to be another factor influencing utilisation of healthcare service in another review, ${ }^{19}$ but we did not come across any such finding in our review.

\subsection{Strengths and weakness}

As our systematic review considered wider ethnic, cultural and geographic regions, it increases the scope of taking a broader view of the findings. Additionally, we have considered perceptions of men and mothers-in-law to get an extensive representation. Quantitative studies often cannot find the underlying reasons for a particular outcome; nevertheless, our review reduces the gap reported by Cleland ${ }^{25}$ by comprehensively identifying the reasons for almost all the FP related concepts in India. Furthermore, these reasons are explained using the WHO framework for social determinates of health. ${ }^{22}$

Despite commonalities in overarching themes, we cannot apply the findings to population belonging to a higher socio-economic stratum because of paucity of data in this group of people. Almost $68.8 \%$ of India's population reside in villages ${ }^{11}$ therefore, findings can be generalised to a larger population of the country. We paid more importance to the experiences and perceptions of people compared to the methodological issues of the included studies. Additionally, we could not categorise the studies based on study quality as measured using Critical Appraisal Skills Programme checklist (Additional file) therefore, we did not use it while generating the findings of the review. Most of the included studies did not report qualitative methodologies appropriately (e.g., type of qualitative data, details on analysis, positionality and reflexivity of researchers); therefore, we suggest future researchers to follow standard reporting guidelines and report a methodology that is reproducible. Furthermore, due to resource constraint, we restricted the studies to English language.

\subsection{Implications for policy and research}

The ongoing programme (RMNCAH+) focusing on SRH and rights in India to be continued and may consider our findings in revising approach for meeting the needs of target population. The implications of the current review findings are listed below- a. Information, education and counselling/behaviour change communication: Our review perpetuates that many perceptions and practices associated with FP adoption were based on misconceptions and traditional socio-cultural beliefs. Developing contextual behaviour change strategies to address the misconceptions and traditional socio-cultural beliefs ${ }^{11}$ without hurting the cultural and religious sentiments could be good approach to increase the awareness of modern contraceptives and alleviate the psychological fears associated with them. An age-appropriate culturally acceptable sex education along with improvement in access to FP services could help in avoiding unintended and early pregnancy, especially among adolescent and young women. ${ }^{24} \mathrm{~A}$ previous conducted review recommended that counselling is an effective means of increasing the uptake of contraceptive use, especially when combined with antenatal or postnatal care. ${ }^{5}$

b. Edutainment approach: Delivering the key messages related to FP using audio-visuals and a story can be a strong media especially for influencing illiterate population.

c. Focusing on sexual and reproductive health and rights: Interventions directed on enhancing the uptake of FP methods can be improvised by giving importance to the demand-side uptake along with a choicebased approach with focus on SRH and rights.

d. Life planning skills: Context-specific mandatory module on life planning skills consisting of SRH and rights should be included in the school curriculum.

e. Community engagement: There is a need for FP program designed through the engagement of various influential stakeholders such as faith healers, informal healthcare providers, high-risk populations and other influential stakeholders. We highlight the importance of woman's agency in $\mathrm{FP}^{30}$ and to involve community to enhance women empowerment by effective awareness creation and shared responsibility among all the stakeholders.

f. Youth responsibility programmes: Youth in India can be the biggest assets in promoting, propagating, creating awareness and influencing (with the help of modern technology/mediums such as social media) the peers and society in using appropriate FP methods. Creation of youth responsibility programmes is the need of the hour.

g. Healthcare strengthening: There is need to increase the accessibility, availability and affordability of modern contraceptive use especially considering the ongoing Covid-19 pandemic, which has disrupted the entire healthcare system in the country.

h. Human resource and capacity building: India has a vast network of accredited social health activists (also known as ASHA), whose capacity can be improvised to deliver some of the contraceptives e.g., condoms and OCPs to the needy population. ASHA's can also be trained to create awareness about FP. Training and capacity building of healthcare providers, frontline health workers and ASHAs should be carried out periodically. ${ }^{12}$ The government can consider using alternative systems of medicine practitioners, especially in rural regions to deliver FP services.

i. Innovative strategies and research: Our findings suggest that conditional cash transfer (monetary incentives for availing sterilisation) from the government health facility was not a motivating factor for the uptake of sterilisation as the money provided was minimal and was spent on medicines. Therefore, we suggest more research on to identify culturally and economically viable methods to increase the access to safe and voluntary FP as a basic human right. Research development should be a priority for meeting the people's needs especially assessing the effectiveness of contraceptives that have low risk of side-effects and are cost-effective.

j. Addressing other social issues: Eliminating gender bias (SDG 5) cannot be addressed in isolation but, can be endeavoured through designing a comprehensive integrated program. The program could focus on addressing dowry, women unemployment and empowerment, education on SRH and rights among the girls and women and 
strict implementation of policies. There is a need to have focused efforts to strengthen eradication of child marriage in India.

$\mathrm{k}$. Some of the stringent laws to restrict the number of children still persists in India, which should be modified considering the SRH and rights. For instance, under National Food Security Act, pregnant women are eligible to receive food and maternity benefits however, it is restricted to only two children. To restrict population explosion giving perks to the people who follow small family norm may work but, any citizen of India should not be disqualified or excluded from the food benefits on the basis of number of children.

\section{Conclusion}

Current review ascertained several barriers related to family planning in India that have yet to be adequately addressed for socioeconomically backward women. Our findings call for reinvigorated efforts to provide effective and individually, religiously, regionally and culturally appropriate family planning interventions and ultimately support better health and rights for the most vulnerable women and communities in India. We also suggest the need for well-designed family planning intervention engaging women, husband and mother-in-law and other stakeholders to address various contextual factors and necessitates with multifaceted actions to improve family planning access in India. Moreover, findings from the current review may assist policymakers to implement context specific policies or programs giving importance to the perceptions of stakeholders as the one-fits-for-all concept becomes obsolete.

\section{Funding statement}

Nil.

\section{Data availability statement}

All data generated or analysed during this study are included in this published article and its supplementary information files.

\section{CRediT authorship contribution statement}

Shradha S. Parsekar: conceptualised the topic, designed and initiated the systematic review, carried out database search and forward and backward citation checking, screened the title and abstracts for inclusion, carried out full text screening and extracted the data from included studies, Formal analysis, which was cross checked, were involved in interpretation of data, made substantial contributions and comments to subsequent drafts and approved the final version for journal submission. Praveen Hoogar: conceptualised the topic, designed and initiated the systematic review, screened the title and abstracts for inclusion, carried out full text screening and extracted the data from included studies, Formal analysis, which was cross checked, were involved in interpretation of data, made substantial contributions and comments to subsequent drafts and approved the final version for journal submission. Vijay Shree Dhyani: carried out full text screening and extracted the data from included studies, were involved in interpretation of data, made substantial contributions and comments to subsequent drafts and approved the final version for journal submission. Uday Narayan Yadav: were involved in interpretation of data, made substantial contributions and comments to subsequent drafts and approved the final version for journal submission.

\section{Declaration of competing interest}

The authors declare that they have no competing interests.

\section{Acknowledgements}

We would like to acknowledge Dr. Ashwini Pujar, Karnataka Health Promotion Trust, Bengaluru-India, for helping us in screening the titles, abstracts and full texts.

\section{References}

1 Parsekar SS, Pundir P, Bevilacqua V. Reproductive, maternal, Newborn, child and adolescent health and related behaviour change communication strategies in Bangladesh, Nepal and India: a narrative review. Clin. Epidemiol. Global Health. 2020; 8(1):280-286. https://doi.org/10.1016/j.cegh.2019.08.014.

2 Family MOHFW. Planning: Ministry of Health and Family Welfare. Government of India; 2021 [updated February 19, 2021; cited 2021 April 21]. Available from: http $\mathrm{s}: / / \mathrm{nhm}$.gov.in/index1.php?lang=1\&level=2\&sublinkid=821\&lid=222. Accessed April 13, 2021

3 Wang GT. Population control policies and implementations in India. J Sociol Soc Welfare. 2019;7(2):135-144.

4 Slaymaker E, Scott R, Palmer M, et al. Trends in sexual activity and demand for and use of modern contraceptive methods in 74 countries: a retrospective analysis of nationally representative surveys. Lancet Global Health. 2020;8:e567-e579. https:// doi.org/10.1016/S2214-109X(20)30060-7.

5 Cavallaro FL, Benova L, Owolabi OO, et al. A systematic review of the effectiveness of counselling strategies for modern contraceptive methods: what works and what doesn't? BMJ. Sex. Reprod. Health. 2020;46:254-269. https://doi.org/10.1136/ bmjsrh-2019-200377.

6 Williamson LM, Parkes A, Wight D, et al. Limits to modern contraceptive use among young women in developing countries: a systematic review of qualitative research. Reprod Health. 2009;6(1):3. https://doi.org/10.1186/1742-4755-6-3.

7 India. Commitment Maker since 2012: FP2030; 2020. Available from: https://www.fa milyplanning2020.org/india. Accessed April 14, 2021.

8 Singh P, Singh KK, Singh P. Factors explaining the dominion status of female sterilization in India over the past two decades (1992-2016): a multilevel study. PLoS One. 2021;16(3), e0246530. https://doi.org/10.1371/journal.pone.0246530.

9 Tobey E, Jain A, Mozumdar A. The relationship between attitudes towards pregnancy and contraceptive continuation: results from a longitudinal study of married women in India. PLoS One. 2020;15(2), e0229333. https://doi.org/10.1371/journal. pone.0229333.

10 Mozumdar A, Tobey E, Aruldas K, et al. Contraceptive Use Dynamics in India: A Prospective Cohort Study of Modern Reversible Contraceptive Users, Research Report. Population council The Evidence Project; 2020.

11 Muttreja P, Singh S. Family planning in India: the way forward. Indian J Med Res. 2018;148(Suppl 1):S1. https://doi.org/10.4103/ijmr.IJMR_2067_17.

12 Singh S, Shekhar C, Acharya R, et al. The incidence of abortion and unintended pregnancy in India. Lancet Global Health. 2018;6(1):e111-e120.

13 Conde-Agudelo A, Belizán JM. Maternal morbidity and mortality associated with interpregnancy interval: cross sectional study. BMJ. 2000;321(7271):1255-1259. https://doi.org/10.1136/bmj.321.7271.1255.

14 Kassebaum NJ, Bertozzi-Villa A, Coggeshall MS, et al. Global, regional, and national levels and causes of maternal mortality during 1990-2013: a systematic analysis for the Global Burden of Disease Study 2013. Lancet. 2014;384(9947):980-1004.

15 Swaminathan A, Fell DB, Regan A, et al. Association between interpregnancy interval and subsequent stillbirth in 58 low-income and middle-income countries: a retrospective analysis using Demographic and Health Surveys. Lancet Global health. 2020;8(1):e113-e122. https://doi.org/10.1016/s2214-109x(19)30458-9.

16 Baxter S, Blank L, Guillaume L, et al. Views of contraceptive service delivery to young people in the UK: a systematic review and thematic synthesis. $J$ Fam Plann Reprod Health Care. 2011;37(2):71. https://doi.org/10.1136/jfprhc. 2010.0014.

17 Rehnström Loi U, Gemzell-Danielsson K, Faxelid E, et al. Health care providers' perceptions of and attitudes towards induced abortions in sub-Saharan Africa and Southeast Asia: a systematic literature review of qualitative and quantitative data. BMC Publ Health. 2015;15(1):139. https://doi.org/10.1186/s12889-015-1502-2.

18 Poels M, Koster MP, Boeije HR, et al. Why do women not use preconception care? A systematic review on barriers and facilitators. Obstet Gynecol Surv. 2016;71(10): 603-612. https://doi.org/10.1097/ogx.0000000000000360.

19 Roberts A, Noyes J. Contraception and women over 40 years of age: mixed-method systematic review. J Adv Nurs. 2009;65(6):1155-1170. https://doi.org/10.1111/ j.1365-2648.2009.04976.x.

20 Atkins S, Lewin S, Smith $\mathrm{H}$, et al. Conducting a meta-ethnography of qualitative literature: lessons learnt. BMC Med Res Methodol. 2008;8(1):21. https://doi.org/ 10.1186/1471-2288-8-21.

21 Thomas J, Harden A. Methods for the thematic synthesis of qualitative research in systematic reviews. BMC Med Res Methodol. 2008;8(1):45. https://doi.org/10.1186/ 1471-2288-8-45.

22 Solar O, Irwin A. A Conceptual Framework for Action on the Social Determinants of Health Social Determinants of Health Discussion Paper 2 (Policy and Practice). Geneva: World Health Organization; 2010.

23 Centre for Child Rights. Handbook on the Prohibition of Child Marriage Act. New Delhi: Ministry of Women and Child Development, Unicef India Country Office; 2006.

24 Ibarra-Nava I, Choudhry V, Agardh A. Desire to delay the first childbirth among young, married women in India: a cross-sectional study based on national survey data. BMC Publ Health. 2020;20(1):350. https://doi.org/10.1186/s12889-020-84029. 
25 Cleland J. The complex relationship between contraception and abortion. Best Pract Res Clin Obstet Gynaecol. 2020;62(2020):90-100. https://doi.org/10.1016/j. bpobgyn.2019.04.007.

26 Starbird E, Norton M, Marcus R. Investing in family planning: key to achieving the sustainable development goals. Glob. Health. Sci. Pract. 2016;4(2):191-210. https:// doi.org/10.9745/GHSP-D-15-00374.

27 Yadav UN, Hosseinzadeh H, Lloyd J, et al. How health literacy and patient activation play their own unique role in self-management of chronic obstructive pulmonary disease (COPD)? Chron Respir Dis. 2019;16. https://doi.org/10.1177/ 1479973118816418.

28 Kaur C. Chhattisgarh HC quashes 1979 order that restricted family planning. Indiana. 2018 December 14:2018.

29 Janis IL, Mann L. Decision Making: A Psychological Analysis of Conflict, Choice, and Commitment. Free press; 1977.

30 Edmeades J, Mejia C, Parsons J, et al. A Conceptual Framework for Reproductive Empowerment: Empowering Individuals and Couples to Improve Their Health (Background Paper). Washington D.C.: International Center for Research on Women.; 2018. 